\title{
THE DYNAMICS OF A NEUROMUSCULAR SYSTEM FUNCTIONAL CONDITION DURING THE PREPARATION FOR IMPORTANT CONTESTS
}

\author{
G. TITOV, M.D. \\ U.S.S.R.
}

\begin{abstract}
The aim of this research was to determine a conjugation of the functional condition dynamics of the neuromuscular organs, visual analyser, latent period of the motorial activity and supporting kinosthetic functions at different stages of preparation of the sportsmen for important contests.

The following research methods were used to achieve this aim: a functional mobility of the neuromuscular organs was determined with the help of an electrostimulation method (excitability thresholds, frequency range of optimum and maximum rhythm, data on the changes of a bioelectric potential in relation to electrical stimuli); a functional condition of the visual analyser was determined with the use of an electrostimulation method (excitability thresholds, frequency range of phosefan in the reaction to a threshold irritant); a grey matter neurodynamics was determined with the help of a chronoreflexometry using an audio irritant of a different power with the preliminary strain of motor centres or without it; supporting kinosthetic functions were determined with the help of seismotremography and stabilography (frequency and amplitude of tremor, deviations of the gravity centre of the body in different positions of the Romberg test).
\end{abstract}

All the research on the systems mentioned was carried out within the preparatory, main and contest periods during the preparation for the important contests. Gymnasts, boxers and fencers were under observation. In all there were carried out $\mathbf{5 7 0}$ observations of $\mathbf{5 4}$ sportsmen of a high sporting qualification.

The functional condition of the visual analyser at a satisfactory level of training was characterised by relatively low excitability thresholds and high frequency limits of phosefan. The functional mobility of the neuromuscular organs was reduced during this period. The supporting kinosthetic functions became apparent in the instability of frequency and amplitude tremor characteristics and general gravity centre deviations. The latent period of the motor movement reaction was the longest without the preliminary strain of the motor centres. The performance of intense training efforts was accompanied by distinct symptoms of the nervous system excitement against a background of the reduced functicnal mobility of the neuromuscular organs.

Just before the main contests, when the sportsmen were in good training condition, the excitability thresholds of the visual analyser were slightly increasing; frequency limits of phosefan were falling; functional mobility of the neuromuscular organs achieved the highest value; supporting kinosthetic functions were characterised by stable values of the tremor frequency and amplitude and of the deviations of the general gravity centre of the body; minimum latent period of the motor movement reaction was dependent upon the preliminary strain of the motor centres.

The data obtained gave us the opportunity to assume that the functional condition dynamics of the systems under consideration might characterise the level of an operative rest, as A. A. Ukhtomsky saw it, as a combatant readiness for physical activity. 